ARTICLE OPEN

\title{
Annular flexible thermoelectric devices with integrated-module architecture
}

\author{
Dawei $\mathrm{Qu}^{1,2}$, Xuan Huang ${ }^{1}$, Xin $\mathrm{Li}^{2 *}$, Hanfu Wang ${ }^{3 *}$ and Guangming Chen (D) $^{1 *}$
}

Organic and composite thermoelectric (TE) materials have witnessed explosive developments in recent years. Design strategy of their flexible devices is vital to achieve high performance and suit various application environments. Here, we propose a design strategy of annular flexible TE devices with integrated-module architecture, where the independent modules made up of alternatively connected p-n couples are connected in series, and then rounded head-to-tail into annular configuration. The achieved devices can not only save plenty of space owing to their highly integrated structure design, but also be directly mounted on cylindrical objects (like pipes) to suit versatile applications. More importantly, the annular TE devices display excellent performances, superior to most previous work and the traditional serial single-layer film structure. For example, the annular device with eight modules consisting of three $\mathrm{p}-\mathrm{n}$ couples reveals an output power of $12.37 \mu \mathrm{W}$ at a temperature gradient of $18 \mathrm{~K}$, much higher than that of the corresponding single-layer film structure $(1.74 \mu \mathrm{W})$. The integration process is simple and easy to scale up. This architecture design strategy will greatly speed up the TE applications and benefit the research of organic and composite TE materials.

npj Flexible Electronics (2020)4:1 ; https://doi.org/10.1038/s41528-020-0064-2

\section{INTRODUCTION}

In all energy materials, thermoelectric (TE) materials are unique and irreplaceable in their capability of harvesting waste or lowquality heat, which covers most of the heat generated in our industrial productions and daily lives. ${ }^{1-7}$ In recent few years, organic and their composite TE materials have witnessed explosive developments owing to their obvious advantages of solution-processability, light-weight, and well source in earth, etc., compared with their conventional inorganic counterparts. ${ }^{8-23}$ For example, various preparation strategies have been developed to achieve organic materials or composites with high TE performances, including template-directed in situ polymerization, nanostructure-controlled construction and layer-by-layer assembly. ${ }^{8-16}$ Indeed, high figure of merit $(Z T)$ value $(0.58)^{17}$ or power factor (PF, $\left.2710 \mu \mathrm{W} \mathrm{m}{ }^{-1} \mathrm{~K}^{-2}\right){ }^{18}$ being almost comparable to the inorganic TE materials, have already been attained. In sharp contrast with the significant breakthroughs for the material research, the studies of TE devices made up of organic and composite TE materials are still in the beginning stage.

High TE output performance and to suit practical application environments are the main objectives for fabrication of TE devices. Design strategy is vital for realizing high performance for TE devices. $^{5}$ Very recently, we proposed that the assembly strategy followed the sequence of the serial $>$ the folding $>$ the stacking for flexible TE devices composed of $p$ - and n-type film couples. ${ }^{24}$ In order to maximize the output power, the increase of $p-n$ pairs is usually applied at a constant temperature gradient. ${ }^{25}$ However, this will inevitably lead to the enlarged device sizes or dimensions, which take up plenty of space and seriously limit their actual applications. On the other hand, annular flexible TE devices are strongly desired in large-scale applications, because waste heat often occurs on the round surfaces of cylindrical objects such as pipes carrying hot fluids, heat engines, and even human wrists or arms. $^{26,27}$ Therefore, it is urgent to develop new assembly strategy of annular flexible TE devices, which can realize high-output TE performance.

In this communication, we propose an assembly design strategy of integrated-module architecture for annular flexible TE devices. First, flexible TE films were fabricated, where pure single-walled carbon nanotubes (SWCNTs) were employed as the p-type component, and n-type materials were obtained by treating SWCNTs with commercialized poly(3,4-ethylenedioxythiophene): poly(styrenesulfonate) (PEDOT:PSS) and subsequent cetyltrimethyl ammonium bromide (CTAB). Then, a TE module was made by alternatively connecting three pairs of $p-n$ films in series with $\mathrm{Cu}$ foils and silver electrodes and then tightly compacted. Finally, eight independent compacted modules were assembled in series and connected head-to-tail, resulting in an annular flexible TE device with integrated-module architecture. The results clearly show that the output power for the annular integrated device $(12.37 \mu \mathrm{W})$ is greatly higher than that made up of traditional single-layer films without integrated architecture $(1.74 \mu \mathrm{W})$. The main reason is that the integrated-module architecture can accommodate more $p$-n couples occupying the same space.

\section{RESULTS}

Characterizations and TE properties of flexible films

Here, the pristine SWCNT is employed as the p-type material, and the n-type films were obtained by treating SWCNT with a commercialized PEDOT:PSS (PH1000) and subsequent polyethyleneimine (PEI) or CTAB (See Supplementary Information 1 for details). Thus, the p-type films contain the pristine SWCNTs, whereas the n-type films consist of SWCNT, PH1000, and PEI or CTAB (Fig. 1a). An example of the $p$ - or n-type film displayed in Fig. $1 \mathrm{~b}$ clearly shows that the composite films with a diameter of

\footnotetext{
${ }^{1}$ College of Materials Science and Engineering, Shenzhen University, Shenzhen 518055, P. R. China. ${ }^{2}$ Beijing Key Laboratory of Clothing Materials R\&D and Assessments, Beijing Engineering Research Center of Textile Nanofiber, School of Materials Science \& Engineering, Beijing Institute of Fashion Technology, Beijing 100029, P. R. China. ${ }^{3}$ National Center for Nanoscience and Technology of China, Beijing 100190, P. R. China. *email: clylx@bift.edu.cn; wanghf@nanoctr.cn; chengm@szu.edu.cn
} 
A

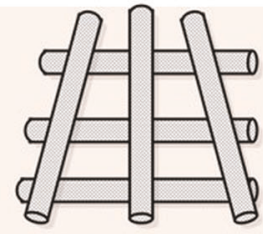

P-type

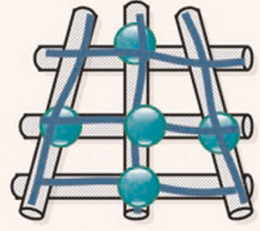

$\mathrm{N}$-type

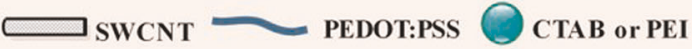

B

a

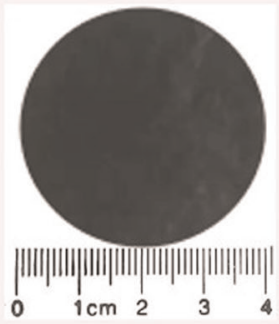

b

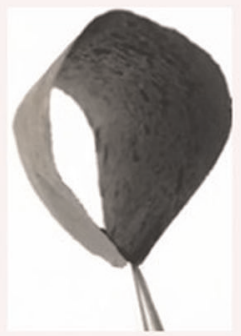

C
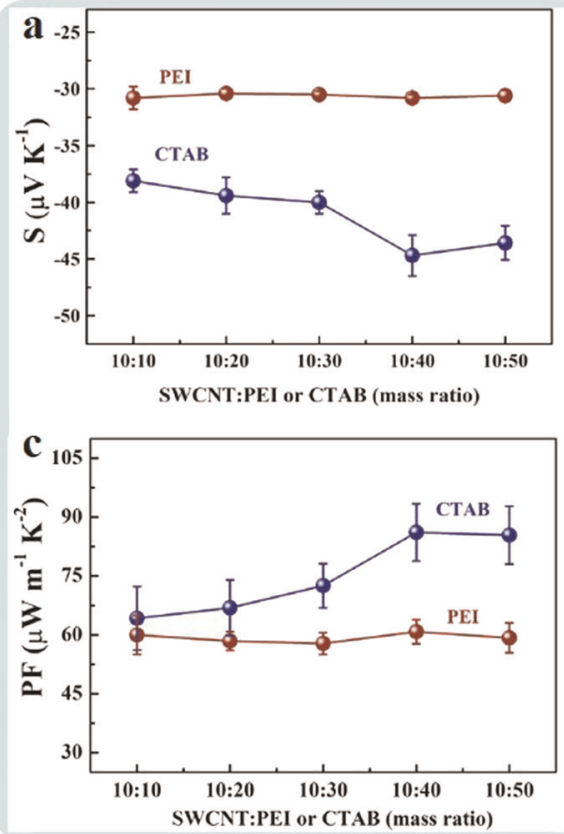

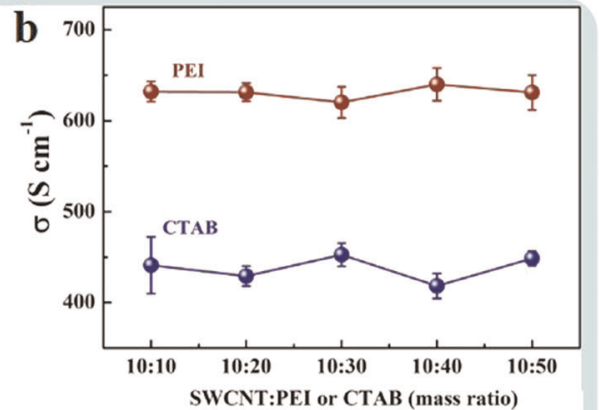

$\mathbf{d}^{2}$

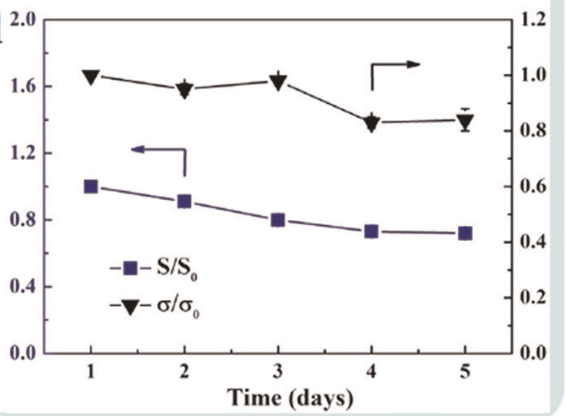

Fig. 1 P- and $\mathbf{n}$-type materials. a Schematic illustration of the $\mathbf{p}$ - and $\mathbf{n}$-type materials. $\mathbf{b}$ Photographs of $\mathbf{a}$ the composite film and $\mathbf{b}$ its high flexibility. c TE performances of the n-type composites including SWCNT/PH1000/PEI and SWCNT/PH1000/CTAB: a Seebeck coefficient, b electric conductivity, and c power factor, where the PH1000 content keeps constant. d The stability test of the SWCNT/PH1000/CTAB composites in air without encapsulation.

$\sim 4 \mathrm{~cm}$ are highly flexible. More detailed information on the morphological and structural characterizations are revealed in Supplementary Figs 1-6. The field-emission scanning electron microscopic (FESEM) observations in Supplementary Fig. 1 shows that the surfaces of the pristine SWCNT bundles are smooth and uniform (a), whereas they become vague and rough for the n-type films in (b) and (c) owing to chemical doping by $\mathrm{PH} 1000$ and PEI or CTAB. Moreover, the results of energy-dispersive X-ray spectroscopy (EDS) (Supplementary Fig. 2) presents that besides the elements of $\mathrm{C}, \mathrm{O}$, and $\mathrm{S}, \mathrm{N}$, and $\mathrm{Br}$ occur in (a), and $\mathrm{N}$ exists in (b), demonstrating the success of the doping by CTAB and PEI in the n-type SWCNT composite films, respectively. In Supplementary Fig. 3a, a strong band at $\sim 1591 \mathrm{~cm}^{-1}$ ( $\mathrm{G}$ band) and a very weak band at $\sim 1346 \mathrm{~cm}^{-1}$ ( $D$ band) occur in the Raman spectra, confirming that after the doping by $\mathrm{PH} 1000$ and $\mathrm{CTAB}$ or PEI, no obvious defects are introduced, and the high crystallinity of the pristine SWCNT is well preserved. Further observation of the radial breathing mode profiles is shown in Supplementary Fig. 3b, characteristic of the out-of-plane stretching phonon mode. The composite curves become smooth and shift a little compared with that for the pristine SWCNT, suggesting that the carbon atoms moving coherently in the radial direction are hindered after doping. ${ }^{28}$ In addition, X-ray photoemission spectroscopy (XPS) was employed to further explore the doping effectiveness. In Supplementary Fig. 4a, a strong peak at $284.4 \mathrm{eV}$ and a weak one at $532 \mathrm{eV}$ are assigned to $\mathrm{C} 1 \mathrm{~s}$ and $\mathrm{O} 1 \mathrm{~s}$, respectively, where the weak $01 \mathrm{~s}$ peak results from the surface defects and the doping of $\mathrm{PH} 1000$. Besides these two peaks, the SWCNT/PH1000/PEI composite displays a strong peak at $399 \mathrm{eV}$, resulting from N1s peak of the PEI doping. Owing to the PH1000 doping, a peak at $167.7 \mathrm{eV}$, characteristic of S2p, takes place in Supplementary Fig. $4 \mathrm{~b}$. In the inset image, the peak of $(68.6 \mathrm{eV})$ can be attributed 
a
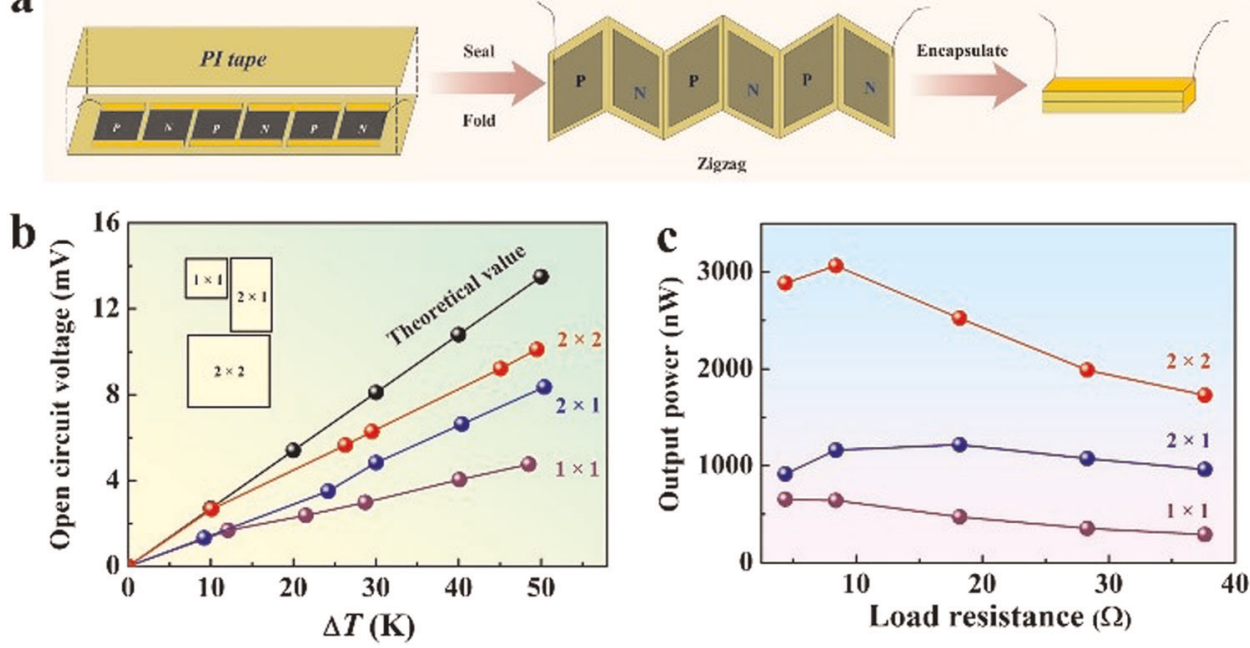

Fig. 2 An individual TE module. a Schematic illustration of the fabrication procedure of a TE module composed of three $p$-n couples with different film sizes $\left(1 \times 1 \mathrm{~cm}^{2}, 2 \times 1 \mathrm{~cm}^{2}\right.$, and $\left.2 \times 2 \mathrm{~cm}^{2}\right)$. b Dependence of open circuit voltage with temperature gradient $(\Delta T)$ for the theoretical values and the measured data of a TE module. $\mathrm{c}$ Effect of the module load resistance on the output power at $\Delta T=45 \mathrm{~K}$.

to $\mathrm{Br} 3 \mathrm{~d}$, resulting from the $\mathrm{CTAB}$ doping. The $\mathrm{X}$-ray diffraction (XRD) patterns in Supplementary Fig. 5 reveal that the composite spectra are almost the same as that of the pristine SWCNT. This demonstrates that the crystalline structure of the pristine SWCNT is not changed after the organic doping, and no excess doping agents exist in the composites. Considering the existence of the $\mathrm{PH} 1000$ and $\mathrm{CTAB}$ or PEI molecules demonstrated by EDS (Supplementary Fig. 2) and XPS spectra (Supplementary Fig. 4), it can be concluded that the organic doping agents are well absorbed on the SWCNT surfaces contributing to the enhanced TE performance.

In Fig. 1c, the composite TE performance (including the Seebeck coefficients (S), the electrical conductivities $(\sigma)$ and the PF (PF $=$ $\left.\left.S^{2} \sigma\right)\right)$ and their stability are displayed. P-type SWCNTs $(S \approx 45 \mu \mathrm{V}$ $\mathrm{K}^{-1}$ ) can be changed into n-type ones by effective charge transferring process of electron donation using a variety of chemicals, such as $\mathrm{PEI}^{28}$ and CTAB. ${ }^{29}$ In (a), the SWCNT/PH1000/ CTAB and the SWCNT/PH1000/PEI composites exhibit negative Seebeck coefficient, confirming the that the main charge carriers are the electrons (characteristic of n-type) rather than the holes (the p-type characteristic). In addition, the absolute values of the composite Seebeck coefficients are almost independent of the mass ratio of SWCNT:PEI, maintaining around $-31 \mu \mathrm{V} \mathrm{K}^{-1}$. As for the SWCNT/PH1000/CTAB composites, they increase greatly first and then reduce with SWCNT:CTAB mass ratio. Moreover, CTAB is superior to PEI in n-type doping with larger Seebeck coefficients (the maximum is $-44.7 \pm 1.8 \mu \mathrm{V} \mathrm{K}^{-1}$ ). In contrast, the composite electrical conductivities shown in (b) are nearly independent of the PEI or CTAB loadings, being $631 \pm 9 \mathrm{~S} \mathrm{~cm}^{-1}$ and $438 \pm 14 \mathrm{~S}$ $\mathrm{cm}^{-1}$, respectively, for those doped by $\mathrm{PEI}$ or $C T A B$ molecules. Consequently, the PFs of CTAB-doped composites increase obviously first and subsequently remain almost constant, with a maximum value of $86.1 \pm 7.3 \mu \mathrm{W} \mathrm{m}{ }^{-1} \mathrm{~K}^{-2}$. The SWCNT/PH1000/PEI composites show a relatively unchanged PF of $\sim 60.0 \pm 5.0 \mu \mathrm{W}$ $\mathrm{m}^{-1} \mathrm{~K}^{-2}$. Because CTAB doping leads to higher TE performances with higher PFs, SWCNT/PH1000/ CTAB composite is chosen to serve as the $n$-type films here in the subsequent fabrication of the flexible TE devices. Figure $1 d$ displays the stability test of the SWCNT/PH1000/CTAB composite in air without encapsulation, where $S_{0}, \sigma_{0}$, and $S, \sigma$ stand for the Seebeck coefficients and the electrical conductivities before and after being kept in air for a certain period, respectively. Distinctly, with the elapse of time, $S / S_{\text {o }}$ and $\sigma / \sigma_{\mathrm{o}}$ decrease gradually to some degree, owing to oxygen doping in air. Indeed, after 5 days, 72 and $84 \%$ of the original values are maintained for the Seebeck coefficient and electrical conductivity of the composites, respectively. In order to avoid significant reduction of the composite TE performance, encapsulation of the TE devices by polyimide (PI) films has been employed in the present study to prevent possible oxygen effect in air.

\section{Preparation and performance of flexible TE modules}

Figure 2 presents (a) a schematic illustration of the fabrication procedure, and ( $b$ and $c$ ) the TE performance of an independent module. The fabrication details are described in the Supporting Information 1. In brief, as shown in Fig. $2 \mathrm{a}$, three pairs of $\mathrm{p}$ - and $\mathrm{n}$ type rectangular films with a desired dimension of $1 \times 1 \mathrm{~cm}^{2}, 2 \times$ $1 \mathrm{~cm}^{2}$, or $2 \times 2 \mathrm{~cm}^{2}$ are alternately connected in series with Cu foils and silver paste, and then encapsulated by PI tapes. Subsequently, they are sealed and folded into zigzag shape. Finally, an independent TE module is obtained after encapsulation. The flexibility was revealed in Supplementary Fig. 6, and the folding processes of the TE modules made up of $p$-n films with different sizes are displayed in Supplementary Fig. 7. Figure $2 \mathrm{~b}$ shows the dependence of theoretical $\left(V_{\mathrm{TH}}\right)$ and actual $\left(V_{\mathrm{AC}}\right)$ open circuit voltage with temperature difference $(\Delta T)$ for the modules with different sizes. The measuring instrument of a TE module is displayed in Supplementary Fig. 8, where the temperature difference across the module is kept constant $(0-50 \mathrm{~K})$. The $V_{\mathrm{TH}}$ can be predicted by:

$V_{T H}=N\left(S_{n}+S_{p}\right) \times \Delta T$

where $S_{n}$ and $S_{p}$ are the Seebeck coefficients of the $n$ - and p-type TE films, respectively. Here, $N$ equals 3 for three $p-n$ pairs. Thus, $V_{\mathrm{TH}}$ increases linearly with increasing $\Delta T$. In Fig. $2 \mathrm{~b}$, the $V_{\mathrm{AC}}$ for all modules increase with the increase of $\Delta T$, but not proportionally like $V_{T H}$. Furthermore, at the same $\Delta T, V_{T H}$ is larger than $V_{A C}$; and $V_{\mathrm{AC}}$ follows the sequence for the films with the dimension of $2 \times$ $2 \mathrm{~cm}^{2}>2 \times 1 \mathrm{~cm}^{2}>1 \times 1 \mathrm{~cm}^{2}$. The maximum $V_{\mathrm{AC}}$ can reach $\sim 10.1 \mathrm{mV}$ at $\Delta T=49.5 \mathrm{~K}$. To have a visual observation of the effect of film dimension on the $V_{\mathrm{AC}}$ of a flexible TE module, a fingertip-touch test is employed at a low $\Delta T(\sim 3 \mathrm{~K})$. As shown in Supplementary Fig. 9, the three modules with the film sizes of $2 \times$ $2 \mathrm{~cm}^{2}, 2 \times 1 \mathrm{~cm}^{2}$, and $1 \times 1 \mathrm{~cm}^{2}$ possess the $V_{A C}$ of $0.8,0.7$, and $0.6 \mathrm{mV}$, respectively, in the fingertip-touch tests, confirming the same order in Fig. $2 b$.

Besides open circuit voltage, output power $(P)$ is critical to the practical applications of TE devices. Generally, the $P$ is described 

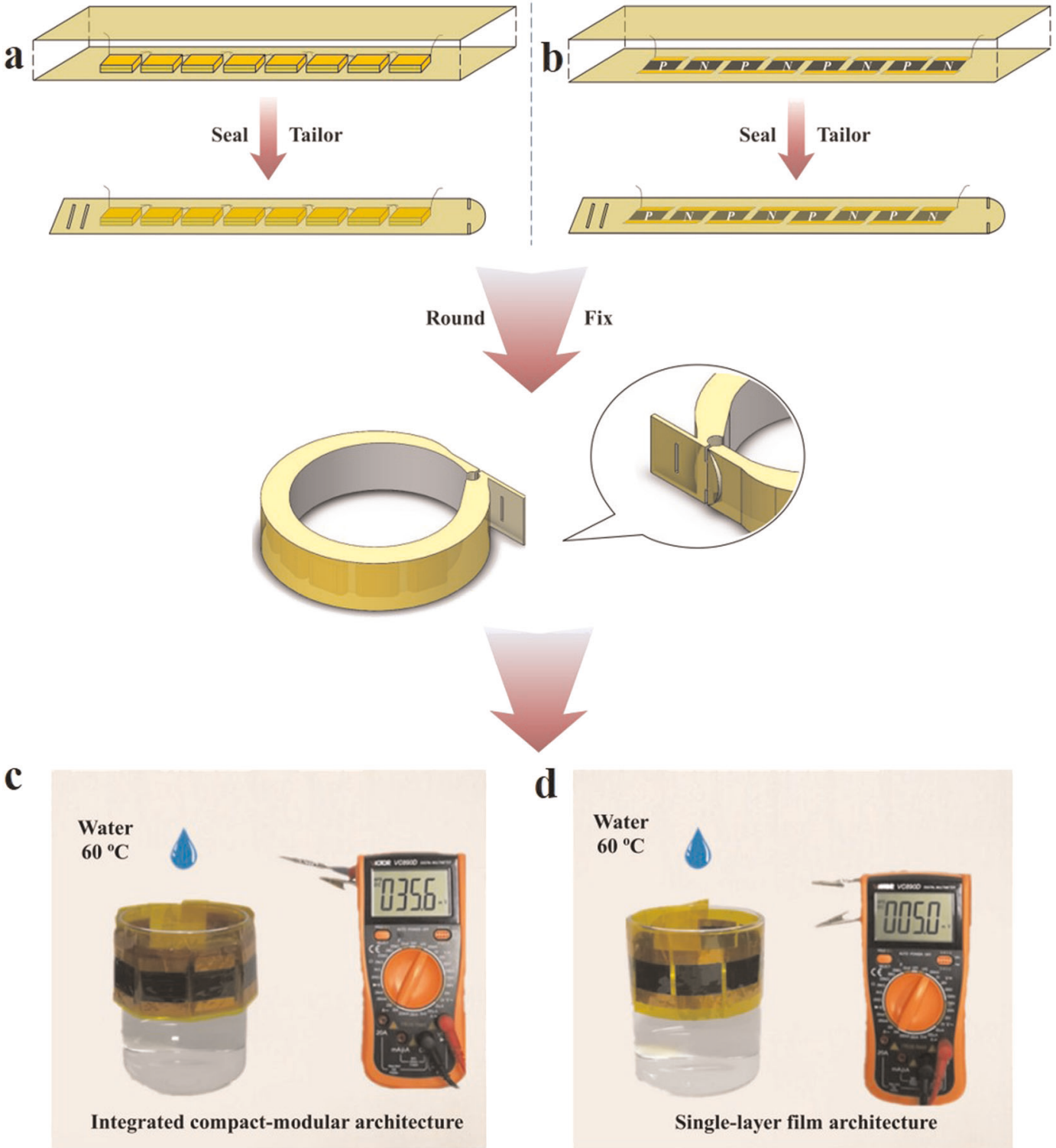

Fig. 3 Schematic illustration of the fabrication procedure of the annular TE devices. a with integrated-module architecture by connecting in series with eight independent modules, each containing three $\mathrm{p}-\mathrm{n}$ couples of single-layer films with a dimension of $2 \times 2 \mathrm{~cm}^{2}, \mathbf{b}$ with singlelayer film structure by alternatively connecting in series using four pairs of the $\mathrm{p}$ - and $\mathrm{n}$-type films with a dimension of $2 \times 2 \mathrm{~cm}^{2}$. $\mathbf{c}$, d Photographs showing the output voltages of the annular TE devices at $\Delta T=18 \pm 2 \mathrm{~K}$ for $\mathbf{c}$ the integrated-module architecture or $\mathbf{d}$ the singlelayer film structure.

by the following equation:

$P=\left[V /\left(R_{\mathrm{r}}+R_{\mathrm{L}}\right)\right]^{2} R_{\mathrm{L}}$

where $R_{\mathrm{r}}$ and $R_{\mathrm{L}}$ represent the intrinsic internal resistance and the load resistance, respectively. Therefore, $P$ reaches its maximum when $R_{\mathrm{r}}$ equals $R_{\mathrm{L}}$ at a constant $\Delta T$. In order to optimize the maximum $P$, the generated current $\left(I_{c}\right)$ and the load circuit voltage $\left(V_{\mathrm{L}}\right)$ from the TE modules are measured as well (Supplementary Fig. 10). Figure 2c clearly shows that the $P$ follows the same sequence to $V_{\mathrm{AC}}$ in Fig. $2 \mathrm{~b}$ at fixed $\Delta T$. When $\Delta T$ is $45 \mathrm{~K}, P$ reaches the maximum of $3065 \mathrm{nW}(8.4 \Omega), 1217 \mathrm{nW}(18.2 \Omega)$, and $654 \mathrm{nW}$ $(4.4 \Omega)$ for the modules with film dimensions of $2 \times 2 \mathrm{~cm}^{2}, 2 \times$ $1 \mathrm{~cm}^{2}$, and $1 \times 1 \mathrm{~cm}^{2}$, respectively. Apparently, large film dimensions lead to higher TE performance at a certain $\Delta T$. However, in the actual applications for the flexible TE devices, a large size is usually not permitted owing to the limited space. As a consequence, an equilibrium between the film dimension and the output performance at a low $\Delta T$ should be considered. Indeed,
Supplementary Fig. 11 shows that even at a small $\Delta T$ of only $6 \mathrm{~K}$, the TE module with the dimension of $2 \times 2 \mathrm{~cm}^{2}$ can reach a higher maximum $P$ of $96 \mathrm{nW}(8.4 \Omega)$ than either of the other modules using smaller p-n films. Considering the results of the $V_{\mathrm{AC}}$ and $P$ shown in Fig. $2 b, c$, the film dimension of $2 \times 2 \mathrm{~cm}^{2}$ is chosen to assemble the TE modules and the subsequent devices. Furthermore, the effect of the pairs of the $p-n$ couples on the module TE performances (open circuit voltages and output powers) are studied, by fixing the dimensions of the films $\left(2 \times 2 \mathrm{~cm}^{2}\right)$. Supplementary Fig. 12 presents three TE modules, consisting of one pair, three and five pairs of the $p-n$ couples $\left(2 \times 2 \mathrm{~cm}^{2}\right)$. The corresponding measured TE properties are shown in Supplementary Fig. 13. In Supplementary Fig. 13a, with the increase of $\Delta T$, all of the open circuit voltages increase greatly, and the sequence follows five pairs $>$ three pairs $>$ one pair. The maximum value reaches $18.5 \mathrm{mV}$ for the module made of five pairs of $\mathrm{p}$-n couples at $\Delta T=50.7 \mathrm{~K}$. Supplementary Fig. $13 \mathrm{~b}$ reveals that at a constant $\Delta T$ of $45 \mathrm{~K}$, the sequence for the output power is consistent with 


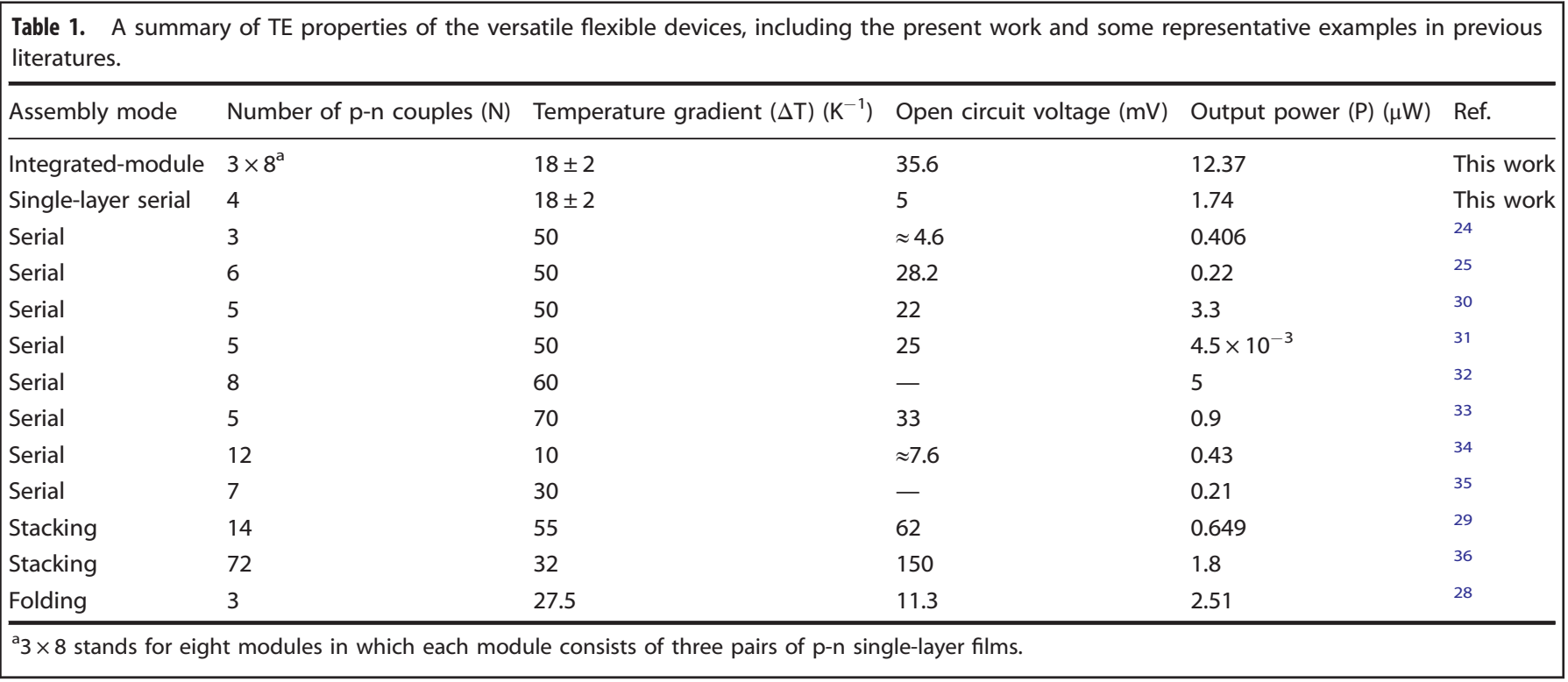

the open circuit voltages, i.e., five pairs $(4721 \mathrm{nW})>$ three pairs $(3065 \mathrm{nW})>$ one pair $(1240 \mathrm{nW})$. Therefore, we conclude that the output TE performance of an independent module strongly depends on the $p-n$ film size, the pair of $p-n$ couples and the $\Delta T$. Large film dimensions, increased pairs of $p-n$ couples and enhanced $\Delta T$ favor the enhanced module TE performances, i.e., higher $V_{\mathrm{AC}}$ and $P$. Considering the actual applications (more $\mathrm{p}-\mathrm{n}$ pairs leading to increased module thickness) and the preparative process, herein, three pairs of $p-n$ couples with film dimension of $2 \times 2 \mathrm{~cm}^{2}$ are applied in the following fabrications of the TE modules and the subsequent devices.

\section{Annular flexible TE device}

The ultimate aim of TE materials is to fabricate their devices for applications in TE generation or refrigeration. Figure 3 presents two kinds of the annular devices with (a) an integrated-module architecture using independent TE modules and (b) traditional serially connected single-layer film assembly. In order to effectively compare the TE performance of these two kinds of annular devices, here, the same $p-n$ materials and the same film dimension of $2 \times 2 \mathrm{~cm}^{2}$ are applied in the device fabrications. Figure $3 a$ displays the fabrication procedure of annular device with an integrated-module structure. In brief, eight independent modules, each consisting of three pairs of $p$-n couples, are first connected in series. Then, by sealing, tailoring, rounding, and fixing, a self-standing and flexible annular device with an integrated-module structure is fabricated. Note that the ends of the annular device do not need encapsulation with tapes owing to a rational tailoring and insertion. Similarly, an annular TE device with traditional single-layer film architecture is obtained (Fig. 3b), where the $\mathrm{p}$ - and n-type single-layer films are alternatively connected in series with $\mathrm{Cu}$ foils and silver paste. The main deviance lies in the independent modules (a) and single-layer films (b). The optical photographs of the two kinds of annular devices are clearly revealed in Supplementary Fig. 14. First, the resistances of the annular devices are measured by a multimeter (Supplementary Fig. 15). The device with an integrated-module structure has a higher resistance of $102.4 \Omega$ than that with the traditional single-layer film architecture (14.4 $\Omega$ ). Then, the TE performance of the two annular TE devices are compared, as shown in Fig. 3c, d. Both devices are tightly mounted on the outer surfaces of cylindrical beakers, and wire leads are connected with a multimeter. After hot water at $60^{\circ} \mathrm{C}$ is poured into the beakers until reaching the lower end of the annular device, the integratedmodular device reveals a higher voltage of $35.6 \mathrm{mV}$ (Fig. 3c), 7.12 times of that of the traditional single-layer film device $(5.0 \mathrm{mV})$ (Fig. 3d). Furthermore, the output powers of the two annular devices are calculated to be 12.37 and $1.74 \mu \mathrm{W}$, respectively. In other words, the integrated-module architecture is much superior to the traditional single-layer film design in output performance for flexible TE devices. Detailed information on recording the voltage change during the pouring process of hot water is distinctly shown in Supplementary Video 1.

In order to compare the present study with the previous literatures, the actual temperature gradient should be measured. We note that in the present study, the hot (lower) end of the devices is $60^{\circ} \mathrm{C}$, whereas the temperature of the cold (upper) end of the devices is not the environmental temperature owing to the presence of hot water vapor. Here, the temperature of the cold (upper) end is measured by an infrared electronic thermometer gun. The corresponding data error is estimated by comparing the above values with the calculated values according to Fig. $2 \mathrm{~b}$ and formula (1) based on the open circuit voltages. Hence, the actual temperature gradient of the annular devices is $18 \pm 2 \mathrm{~K}$. Table 1 illustrates a summary of the performance for TE devices, including the present work and some representative examples in previous literatures. Obviously, the annular device with integrated-module structure reported here displays excellent TE performance with high open circuit voltage and output power at a small temperature gradient of $18 \mathrm{~K}$. One main reason is its integratedmodule architecture that it can accommodate more $p-n$ couples when occupying the same space.

\section{DISCUSSION}

For applications, ideal TE devices should possess reasonable shapes to suit their applied environments and excellent output performances. Annular flexible TE devices are strongly desired in large-scale applications, because waste or low-quality heat often occurs on the round surfaces of cylindrical objects such as pipes carrying hot fluids, heat engines, and even human wrists or arms. On the other hand, design strategy is vital for realizing high performance for these flexible TE devices. Very recently, we proposed that the assembly strategy followed the sequence of serial $>$ folding $>$ stacking for flexible TE devices composed of $\mathrm{p}$ - and n-type film couples. ${ }^{24}$ In order to optimize the output power, the increase of $p-n$ pairs is usually applied. However, this 
will inevitably lead to the enlarged device sizes or dimensions, which take up plenty of space and seriously limits their applications. Therefore, it is urgent to develop new assembly strategy of annular flexible TE devices with high-output TE performance.

Here, we have proposed a design strategy to fabricate flexible TE device, i.e., annular device with integrated-module architecture, which generates an output power of $12.37 \mu \mathrm{W}$ at a small temperature gradient of $18 \mathrm{~K}$. Its TE performance is obviously superior to most previous work and the corresponding annular device with the conventional single-layer film structure $(1.74 \mu \mathrm{W})$. The judicious annular design can significantly widen the application environments such as mounting on cylindrical objects like pipes, owing to the self-standing and flexibility characteristics. Furthermore, the unique integrated-module architecture can accommodate more $\mathrm{p}$-n couples occupying the same space, and thus display higher TE output performance. The integration process is simple and easy to scale up. And the film size, the p$\mathrm{n}$ pairs and the module number can be adjusted and optimized according to the application conditions. We believe that our research is a significant advance in assembly design and actual application of flexible TE devices, which will benefit the developments of TE materials and speed up the versatile applications of waste heat harvesting, wireless sensor networks, e-skin, and wearable equipment, etc.

\section{METHODS}

\section{Materials}

Commercialized SWCNTs (NTP SWNT-2) with a diameter $<3 \mathrm{~nm}$ and a purity $>85.0$ wt $\%$ were bought from Shenzhen Nanotech Port Co. Ltd., China. CLEVIOS PH1000 was purchased from Heraeus Deutschland Gmbh. Dimethylformamide $\left(\mathrm{C}_{3} \mathrm{H}_{7} \mathrm{NO}\right.$, A.R.) was purchased from Mackiln, whereas CTAB $\left(\mathrm{C}_{16} \mathrm{H}_{33}\left(\mathrm{CH}_{3}\right)_{3} \mathrm{NBr}\right.$, > 99\% in purity), branched PEl (molecular weight: $600,99 \%)$ and anhydrous ethanol (A.R.) were bought from Aladdin company.

\section{Preparation of $\mathrm{p}$ - and $\mathrm{n}$-type films}

First, $10 \mathrm{mg}$ of SWCNT was dispersed into $25 \mathrm{~mL}$ of ethanol, and ultrasonically treated in ice bath for $30 \mathrm{~min}$. Later, the mixture was vacuum-filtered on a porous nylon membrane, and then dried under vacuum at $60^{\circ} \mathrm{C}$ for $1 \mathrm{~h}$, affording the $\mathrm{p}$-type SWCNT film. As for the $\mathrm{n}$-type composite film, $10 \mathrm{mg}$ of SWCNT was added into $25 \mathrm{~mL}$ of DMF solution containing $100 \mathrm{~L}$ of $\mathrm{PH} 1000$ and a desired amount of CTAB or PEl, namely, $10,20,30,40$, or $50 \mathrm{mg}$, and ultrasonically treated in ice bath for $30 \mathrm{~min}$. Similarly, the mixture solution was vacuum-filtrated with a porous nylon membrane to obtain a composite film with $25 \mu \mathrm{m}$ in thickness, and finally dried under vacuum at $60^{\circ} \mathrm{C}$ for $1 \mathrm{~h}$.

\section{Fabrication of the serial modules}

The as-prepared $\mathrm{p}$ - and $\mathrm{n}$-type films were cut into rectangular ribbons with a desired dimension of $1 \times 1,2 \times 1$, or $2 \times 2 \mathrm{~cm}^{2}$. The fabrication process is shown in Fig. 2a. First, three pairs of $p$ - and n-type films were alternatively connected in series using copper foil and silver electrodes on a PI film substrate. Then, two copper wires were embedded into the two ends of the $p$ - and n-type films for measuring the output voltage. After that, they were sealed by PI tapes in order to avoid air contact and oxygen doping. Finally, the module was folded into zigzag shape and encapsulated by PI tapes, providing a serial module. Similarly, the modules with the different film sizes, including $2 \times 1$ and $2 \times 2 \mathrm{~cm}^{2}$, were also assembled using the above mode. In addition, to make comparison, the module containing one pair, three or five pairs of $\mathrm{p}$ - and $\mathrm{n}$-type films (Supplementary Fig. 12) were fabricated according to the same procedure. Finally, all of the above modules were dried under vacuum at $60^{\circ} \mathrm{C}$ for $2 \mathrm{~h}$ to ensure the effective curing of the silver paste.

\section{Fabrication of annular devices}

As displayed in Fig. 3a, eight modules consisting of three pairs of $p-n$ couples with the film dimension of $2 \times 2 \mathrm{~cm}^{2}$ were connected in series on the PI substrate, and subsequently sealed by $\mathrm{PI}$ tapes. For convenience of device fixation, one end was tailored into a semicircle shape, whereas the other end was cut into two rectangular holes for insertion. Finally, the integrated compact-modular annular devices were achieved by rounding and fixing (insertion). In Fig. $3 \mathrm{~b}$, four pairs of individual $\mathrm{p}$ - and $\mathrm{n}$-type films with the same dimension $\left(2 \times 2 \mathrm{~cm}^{2}\right)$ were alternatively connected in series using copper foil and silver electrodes on a PI substrate. Then, two copper wires were embedded into the ends of the $\mathrm{p}$ - and n-type films, and they were later sealed by PI tape. Finally, by tailoring, rounding, and fixing procedures in accordance with the above-integrated device fabrication, annular devices of single-layer $p-n$ films were assembled.

\section{Morphology and structural characterizations}

The surface morphology and the corresponding elements' distributions by EDS were directly observed by a FEI APREO S FESEM at an acceleration voltage of $10 \mathrm{kV}$. Raman spectra (RENIDHAW invia Raman Microscope) were recorded within the wavenumber range of $100-4000 \mathrm{~cm}^{-1}$ using a laser diode at an excitation wavelength of $514.5 \mathrm{~nm}$ and a nominal resolution of $4 \mathrm{~cm}^{-1}$. XPS analysis was conducted by a multipurpose XPS (Microlab 350). Powder XRD measurements were carried out using a Bruker D8 Advance at a scanning rate of $10^{\circ} \mathrm{min}^{-1}$.

\section{Measurements of TE performance}

The electrical conductivities and the Seebeck coefficients at room temperature were measured using a Film Thermoelectric Parameter Test System (MRS-3RT, JiaYiTong Company) at a quasi-steady state mode. The resistances and the output voltages of the TE modules were measured by the commercial instruments, Keithley 192 Programmable DMM and a Keithley 2000 Multimeter (Keithley Instruments Inc., USA), respectively. The resistances and the output voltages of the annular devices were measured by a digital multimeter (Double King Industrial Holdings Co., Ltd.).

As displayed in Supplementary Fig. 8, the photograph shows an equipment of testing TE modules, consisting of a hot plate, a cold plate, temperature control systems and a Keithley 2400 sourcemeter. First, the module was placed between the cold plate and the hot plate, noting that one end of the module with wire leads should be put on the cold plate. Later, two pairs of T-type thermocouples were fixed on either side of the module to monitor the temperatures of the hot and the cold ends. The temperature of the module hot end was adjusted by a temperature controller (Lakeshore 336), whereas the temperature of the cold end was maintained at $300 \mathrm{~K}$ using a commercial Peltier module in contact with a circulating water cooler. Then, by controlling the temperature difference $(\Delta T)$ across the module with desired values between 0 and $50 \mathrm{~K}$ and varying the resistance of load resistor $\left(R_{\mathrm{L}}\right)$ connected with the module, the electrical current $\left(I_{C}\right)$ in the circuit was monitored by the Keithley 2400 sourcemeter. The output power $(P)$ was then calculated from the expression $P=l_{\mathrm{C}}^{2} R_{\mathrm{L}}$. The maximum output power can be identified from $P \sim R_{\mathrm{L}}$ plots. As for the open circuit voltage $\left(V_{\mathrm{AC}}\right)$, the TE module was directly connected to the Keithley 2400 sourcemeter by removing the load resistor.

\section{DATA AVAILABILITY}

The authors declare that the data supporting the findings of this study are available within the paper (and its supplementary information files). Data are available upon request.

Received: 25 November 2019; Accepted: 13 January 2020; Published online: 29 January 2020

\section{REFERENCES}

1. Twaha, S., Zhu, J., Yan, Y. \& Li, B. A comprehensive review of thermoelectric technology: materials, applications, modelling and performance improvement. Renew. Sustain. Energy Rev. 65, 698-726 (2016).

2. He, J. \& Tritt, T. M. Advances in thermoelectric materials research: looking back and moving forward. Science 357, 1369 (2017).

3. Siddique, A. R., Mahmud, M. S. \& Heyst, B. V. A review of the state of the science on wearable thermoelectric power generators (TEGs) and their existing challenges. Renew. Sustain. Energy Rev. 73, 730-744 (2017). 
4. Yu, P., Wu, L., Zhou, L. \& Chen, L. Deep-ultraviolet nonlinear optical crystals: $\mathrm{Ba}_{3} \mathrm{P}_{3} \mathrm{O}_{10} \mathrm{X}(\mathrm{X}=\mathrm{Cl}, \mathrm{Br})$. J. Am. Chem. Soc. 136, 480-487 (2014).

5. Blackburn, J. L., Ferguson, A. J., Cho, C. \& Grunlan, J. C. Carbon-nanotube-based thermoelectric materials and devices. Adv. Mater. 30, 1704386 (2018).

6. Liu, Y. et al. The antimony-based type I clathrate compounds $\mathrm{Cs}(8) \mathrm{Cd}(18) \mathrm{Sb}(28)$ and $\mathrm{Cs}(8) \mathrm{Zn}(18) \mathrm{Sb}$ (28). Angew. Chem. Int. Ed. 48, 5305-5308 (2009).

7. Hong, M. et al. Enhancing the thermoelectric performance of SnSe1-xTex nanoplates through band engineering. J. Mater. Chem. A 5, 10713-10721 (2017).

8. Xu, K., Chen, G. \& Qiu, D. Convenient construction of poly(3,4ethylenedioxythiophene)-graphene pie-like structure with enhanced thermoelectric performance. J. Mater. Chem. A 1, 12395-12399 (2013).

9. Moriarty, G. P., Wheeler, J. N., Yu, C. \& Grunlan, J. C. Increasing the thermoelectric power factor of polymer composites using a semiconducting stabilizer for carbon nanotubes. Carbon 50, 885-895 (2012).

10. Hu, X., Chen, G., Wang, X. \& Wang, H. Convenient construction of poly(3,4ethylenedioxythiophene)-graphene pie-like structure with enhanced thermoelectric performance. J. Mater. Chem. A 2, 20896-20902 (2015).

11. Zhang, Z., Chen, G., Wang, H. \& Zhai, W. Enhanced thermoelectric property by the construction of a nanocomposite 3D interconnected architecture consisting of graphene nanolayers sandwiched by polypyrrole nanowires. J. Mater. Chem. C. 3 , 1645-1654 (2015).

12. Hu, X., Chen, G. \& Wang, X. An unusual coral-like morphology for composites of poly(3,4-ethylenedioxythiophene)/carbon nanotube and the enhanced thermoelectric performance. Compos. Sci. Technol. 144, 43-50 (2017).

13. Xue, Y., Gao, C., Liang, L., Wang, X. \& Chen, G. Nanostructure controlled construction of high-performance thermoelectric materials of polymers and their composites. J. Mater. Chem. A 6, 22381-22390 (2018).

14. Han, S., Zhai, W., Chen, G. \& Wang, X. Morphology and thermoelectric properties of graphene nanosheets enwrapped with polypyrrole. RSC Adv. 4, 19218-19220 (2014).

15. Yao, C.-J., Zhang, H.-L. \& Zhang, Q. Recent progress in thermoelectric materials based on conjugated polymers. Polymers 11, 107 (2019).

16. $\mathrm{Wu}$, J., Sun, Y., Xu, W. \& Zhang, Q. Investigating thermoelectric properties of doped polyaniline nanowires. Synth. Met. 189, 177-182 (2014).

17. Wang, L. et al. Exceptional thermoelectric properties of flexible organic-inorganic hybrids with monodispersed and periodic nanophase. Nat. Commun. 9, 3817 (2018)

18. Cho, C. et al. Outstanding low temperature thermoelectric power factor from completely organic thin films enabled by multidimensional conjugated nanomaterials. Adv. Energy Mater. 6, 1502138 (2016).

19. Meng, Q., Jiang, Q., Cai, K. \& Chen, L. Preparation and thermoelectric properties of PEDOT:PSS coated Te nanorod/PEDOT:PSS composite films. Org. Electron. 64 79-85 (2019)

20. Ryan, J. D. et al. All-organic textile thermoelectrics with carbon-nanotube-coated n-type yarns. ACS Appl. Energy Mater. 1, 2934-2941 (2018).

21. Wang, Y. et al. Flexible thermoelectric materials and generators: challenges and innovations. Adv. Mater. 31, 1807916 (2019).

22. Wu, G., Gao, C., Chen, G., Wang, X. \& Wang, H. High-performance organic thermoelectric modules based on flexible films of a novel n-type single-walled carbon nanotube. J. Mater. Chem. A 4, 14187-14193 (2016).

23. Taroni, P. et al. Toward stretchable self-powered sensors based on the thermoelectric response of PEDOT:PSS/polyurethane blends. Adv. Funct. Mater. 28, 1704285 (2018)

24. Qu, D., Li, X., Wang, H. \& Chen, G. Assembly strategy and performance evaluation of flexible thermoelectric devices. Adv. Sci. 6, 1900584 (2019).

25. Song, $\mathrm{H}$. et al. Polymer/carbon nanotube composite materials for flexible thermoelectric power generator. Compos. Sci. Technol. 153, 71-83 (2017).

26. Madan, D. et al. Enhanced performance of dispenser printed MA n-type Bi2Te3 composite thermoelectric generators. ACS Appl. Mater. Interfaces 4, 6117-6124 (2012).

27. Madan, D., Wang, Z., Chen, A., Wright, P. K. \& Evans, J. W. High-performance dispenser printed MA p-type $\mathrm{Bi}(0.5) \mathrm{Sb}(1.5) \mathrm{Te}(3)$ flexible thermoelectric generators for powering wireless sensor networks. ACS Appl. Mater. Interfaces 5 , 11872-11876 (2013).

28. Zhou, W. et al. High-performance and compact-designed flexible thermoelectric modules enabled by a reticulate carbon nanotube architecture. Nat. Commun. $\mathbf{8}$ 14886 (2017).

29. Cheng, X., Wang, X. \& Chen, G. A convenient and highly tunable way to n-type carbon nanotube thermoelectric composite film using common alkylammonium cationic surfactant. J. Mater. Chem. A 6, 19030-19037 (2018).
30. Wu, G. et al. Exploring high-performance n-Type thermoelectric composites using amino-substituted rylene dimides and carbon nanotubes. ACS Nano 11 5746-5752 (2017).

31. Piao, M. et al. Increasing the thermoelectric power generated by composite films using chemically functionalized single-walled carbon nanotubes. Carbon 62 430-437 (2013).

32. Devendra Singh, A. T. K. et al. Strain-induced rolled thin films for lightweight tubular thermoelectric generators. Adv. Mater. Technol. 3, 1700192 (2018).

33. Tian, R. et al. A solution-processed $\mathrm{TiS}_{2}$ /organic hybrid superlattice film towards flexible thermoelectric devices. J. Mater. Chem. A 5, 564-570 (2017).

34. Kim, J.-Y., Lee, W., Kang, Y. H., Cho, S. Y. \& Jang, K.-S. Wet-spinning and posttreatment of CNT/PEDOT:PSS composites for use in organic fiber-based thermoelectric generators. Carbon 133, 293-299 (2018).

35. Takashiri, M., Shirakawa, T., Miyazaki, K. \& Tsukamoto, H. Fabrication and characterization of bismuth-telluride-based alloy thin film thermoelectric generators by flash evaporation method. Sens. Actuator A Phys. 138, 329-334 (2007).

36. Kim, S. L., Choi, K., Tazebay, A. \& Yu, C. Flexible power fabrics made of carbon nanotubes for harvesting thermoelectricity. ACS Nano 8, 2377-2386 (2014).

\section{ACKNOWLEDGEMENTS}

G.C. thanks the National Natural Science Foundation of China (No. 51973122 and 51573190) for financial support. X.L. acknowledges the National Natural Science Foundation of China (No. 51873003), Collaborative Innovation Project in Chaoyang District, Beijing (No. CYXC1718), and Special funds for the construction of high level teachers of Beijing Institute of Fashion Technology (BIFTXZ201802).

\section{AUTHOR CONTRIBUTIONS}

G.C. designed and supervised the project. D.Q. performed the material synthesis and characterization, device fabrication, and testing. H.X. helped the experimental work of film preparation and movie recording. X.L. discussed the results. H.W. helped the design and test of the device. D.Q. and G.C. wrote the paper. All authors provided comments for the manuscript.

\section{COMPETING INTERESTS}

The authors declare no competing interests.

\section{ADDITIONAL INFORMATION}

Supplementary information is available for this paper at https://doi.org/10.1038/ s41528-020-0064-2.

Correspondence and requests for materials should be addressed to X.L., H.W. or G.C.

Reprints and permission information is available at http://www.nature.com/ reprints

Publisher's note Springer Nature remains neutral with regard to jurisdictional claims in published maps and institutional affiliations.

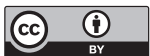

Open Access This article is licensed under a Creative Commons Attribution 4.0 International License, which permits use, sharing, adaptation, distribution and reproduction in any medium or format, as long as you give appropriate credit to the original author(s) and the source, provide a link to the Creative Commons license, and indicate if changes were made. The images or other third party material in this article are included in the article's Creative Commons license, unless indicated otherwise in a credit line to the material. If material is not included in the article's Creative Commons license and your intended use is not permitted by statutory regulation or exceeds the permitted use, you will need to obtain permission directly from the copyright holder. To view a copy of this license, visit http://creativecommons. org/licenses/by/4.0/.

(c) The Author(s) 2020 